\title{
Fixed point iteration for a countable family of multi-valued strictly pseudo-contractive-type mappings
}

\author{
C. E. Chidume ${ }^{1,2}$ and M. E. Okpala $2^{2^{*}}$
}

*Correspondence:

maejok@gmail.com

${ }^{2}$ Department

of Mathematics, Federal University Ndufu-Alike Ikwo,

Abakaliki, Ebonyi State,

Nigeria

Full list of author information

is available at the end of the article

\begin{abstract}
This paper introduces a new averaged algorithm for finding a common fixed point of a countably infinite family of generalized $k$-strictly pseudocontractive multi-valued mappings. The new iterative sequence introduced is proved to be an approximating fixed point sequence for common fixed points of a countably infinite family of this class of mappings. Furthermore, under some mild assumptions, strong convergence theorems are also proved for this class of mappings. The method of proof used here is new and enables to overcome many strong restrictions appearing in contemporary literature.

The stated theorems improve and generalize many recent works in iterative scheme for multi-valued mappings.
\end{abstract}

Keywords: Generalized k-strictly pseudo-contractive multi-valued mappings, Multivalued maps

Mathematics Subject Classification: 47H04, 47H09, 47H10

\section{Background}

Let $(X, d)$ be a metric space, $K$ a nonempty subset of $X$, and $T: K \rightarrow 2^{K}$ be a multivalued mapping. A vector $x \in K$ is a fixed point of $T$ if $x \in T x$. For a single valued mapping $T$, a fixed point is any $x \in K$ such that $T x=x$. We denote the collection of all fixed points of $T$ by $F(T)$. Many well known researchers like Brouwer (1912), Daffer and Kaneko (1995), Deimling (1992), and Kirk Downing and Kirk (1977), Geanakoplos (2003), Kakutani (1941), Markin (1973), Nadler (1969), Nash (1950, 1951) and Reich and Zaslavski (2002a, b, 2006), have studied fixed points for multi-valued mappings.

Fixed point theory for multi-valued mappings continues to attract a lot of attention because of its numerous real world applications in game theory and market economy, differential inclusions, and constrained optimization. They are also desirable in devising critical points in optimal control problems, energy management problems, signal processing, image reconstruction and a host of other problems.

Game theory and market economy is, perhaps, the most socially recognized application of multi-valued mappings.

(C) 2015 Chidume and Okpala. This article is distributed under the terms of the Creative Commons Attribution 4.0 International License (http://creativecommons.org/licenses/by/4.0/), which permits unrestricted use, distribution, and reproduction in any medium, provided you give appropriate credit to the original author(s) and the source, provide a link to the Creative Commons license, and indicate if changes were made. 
Consider, for example, a game $G\left(x_{n}, K_{n}\right)$ involving $N$ players, namely $n=1,2, \ldots, N$. Here, $K_{n}$, a nonempty compact and convex subset of $\mathbb{R}^{m_{n}}$, is the collection of possible strategies of the $n$th player. The continuous function $x_{n}: \Pi_{i=1}^{N} K_{n} \rightarrow \mathbb{R}$, is the gain(payoff) function. Any vector $y_{n}$ in $K_{n}$ is the action which is available to the individual $n$ to take. The collective action of all the $N$ players is then $y:=\left(y_{1}, y_{2}, \ldots, y_{N}\right) \in K:=\Pi_{i=1}^{N} K_{n}$. Given any $n, y$ and $y_{n} \in K_{n}$, we use these standard notations:

$$
\begin{aligned}
K_{-n}: & =K_{1} \times K_{2} \times \cdots \times K_{n-1} \times K_{n+1} \times \cdots \times K_{n} \\
y_{-n}: & =\left(y_{1}, \ldots, y_{n-1}, y_{n+1}, \ldots, y_{N}\right) \\
\left(y_{n}, y_{-n}\right) & =\left(y_{1}, y_{2}, \ldots, y_{n-1}, y_{n}, y_{n+1}, \ldots, y_{N}\right) .
\end{aligned}
$$

In this regard, the $n^{\prime}$ th player maximizes his own gain, using a strategy $y_{n}^{*}$, subject to the fact that the other players have chosen their strategies $y_{-n}$ if and only if

$$
x_{n}\left(y_{n}^{*}, y_{-n}\right)=\max _{y_{n} \in K_{n}} x_{n}\left(y_{n}, y_{-n}\right) \text {. }
$$

Define a multi-valued mapping $T_{n}: K_{-n} \rightarrow 2^{K_{n}}$ by

$$
T_{n}\left(y_{-n}\right)=\operatorname{Arg} \max _{y_{n} \in K_{n}} x_{n}\left(y_{n}, y_{-n}\right)
$$

Then, the collective action $y^{*}=\left(y_{1}^{*}, y_{2}^{*}, \ldots, y_{N}^{*}\right)$ is called a Nash equilibrium point if each $y_{n}^{*}$ is the most effective response that the $n^{\prime}$ th player can make to the actions $y_{-n}^{*}$ of the other $N-1$ players. This is stated differently as

$$
x_{n}\left(y_{n}^{*}\right)=\max _{y_{n} \in K_{n}} x_{n}\left(y_{n}, y_{-n}^{*}\right)
$$

or, in other words,

$$
y_{n}^{*} \in T_{n}\left(y_{-n}^{*}\right) .
$$

Therefore, $y^{*}=\left(y_{1}^{*}, y_{2}^{*}, \ldots, y_{N}^{*}\right)$ is a fixed point of the multi-valued mapping $T: K \rightarrow 2^{K}$ given by

$$
T(y)=\left[T_{1}\left(y_{-1}\right), T_{2}\left(y_{-2}\right), \ldots, T_{N}\left(y_{-N}\right)\right] .
$$

Though many theory for multi-valued mappings in the literature have dealth with the existence of fixed points for such mappings, only very few have dealth with iterative algorithms for computing them. The problem of how to find such fixed points is part of what is addressed in this paper.

Given a real Hilbert space $H$, we denote by $C B(H)$ the family of nonempty, closed and bounded subsets of $H$. It is well known that the Hausdorff distance defined by

$$
D(A, B):=\max \left\{\sup _{a \in A} d(a, B), \sup _{b \in B} d(b, A)\right\}
$$

is a metric on this family $C B(H)$.

The first work on fixed points for multi-valued (nonexpansive) mappings by the application of Hausdorff metric was done by Markin (1973), and followed by an extensive work by Nadler (1969). Since then, there are many results that have appeared in the 
literature and which have found novel applications in both pure and applied sciences. Notable among these results is the work of Browder (1967).

In studying the operator equation $A u=0$ (when the mapping $A$ is monotone), Browder (1967), introduced a new operator $T$ defined by $T:=I-A$, where $I$ is the identity mapping on the Hilbert $H$. He called the operator a pseudocontractive mapping and the solutions of $A u=0$, are exactly the fixed points of the pseudocontractive mapping $T$. An important proper subclass of the pseudocontractive mappings is the well know nonexpansive mappings.

Definition 1.1 A single-valued mapping $T: K \subseteq H \rightarrow H$ is called

- pseudo-contractive, in the terminology of Browder and Petryshyn (1967), if there exists $k \in[0,1)$ such that

$$
\|T x-T y\|^{2} \leq\|x-y\|^{2}+k\|(x-T x)-(y-T y)\|^{2}, \quad \forall x, y \in K .
$$

- monotone if

$$
\langle T x-T y, x-y\rangle \geq 0, \quad \forall x, y \in D(T) .
$$

The class of pseudocontractive mappings is particularly important due to this close connection it has with the well known class of monotone mappings. Fixed points of the pseudocontractive mapping $T$ are zeroes of the monotone mapping $A=I-T$. A well known example of a monotone operator in optimization theory is the multi-valued mapping $\partial f: D(f) \subseteq H \rightarrow 2^{H}$ called the subdifferential of the functional $f$ and defined by

$$
\partial f(x):=\left\{x^{*} \in X^{*}:\left\langle x-y, x^{*}\right\rangle \leq f(x)-f(y), \forall y \in X\right\} .
$$

The theory of multi-valued nonexpansive mappings(and, in particular, pseudocontractive mappings) is much harder than the corresponding theory of single valued nonexpansive mappings [see e.g. Khan and Yildirim (2012)]. The extension of the notion of single valued pseudocontractive mappings to multi-valued pseudocontractive mappings has some of these challenges:

- Definition of the mapping There is a problem of getting a right definition for the multi-valued analogue which would be a generalization of the single-valued case. There are several definitions available which will be a generalisation of the single valued case and one has to get the most natural among them to be able to establish some convergence theorems.

- Identities In multi-valued settings, the metric induced by the norm on $X$ is not applicable and there is the need to develop new identities and other notions of distances which will be applicable. One notion of metric for sets that is readily applicable here is the Hausdorf metric.

- Inference Many theorems and lemmas that are developed for single valued mappings cannot be carried over to multi-valued cases and it is always difficult to make conclusions. 
Chidume et al. (2013), introduced a multi-valued analogue of Definition 1.2 as follows;

Definition 1.2 Let $H$ be a real Hilbert space and let $D$ be a nonempty, open and convex subset of $H$. Let $T: \bar{D} \rightarrow C B(\bar{D})$ be a mapping. Then, $T$ is called a multi-valued $k$-strictly pseudocontractive mapping if there exists $k \in(0,1)$ such that for all $x, y \in D(T)$, we have

$$
D^{2}(T x, T y) \leq\|x-y\|^{2}+k\|(x-u)-(y-v)\|^{2},
$$

for all $u \in T x, v \in T y$.

They proved a convergence theorem for this class of mapping as stated below:

Theorem 1.3 (Chidume et al. (2013)) Let $K$ be a nonempty, closed and convex subset of a real Hilbert space H. Suppose that $T: K \rightarrow C B(K)$ is a multi-valued $k$-strictly pseudocontractive mapping such that $F(T) \neq \emptyset$. Assume that $T p=\{p\}$ for all $p \in F(T)$. Suppose that $T$ is hemicompact and continuous. Let $\left\{x_{n}\right\}$ be a sequence defined iteratively from $x_{0} \in K$ by

$$
x_{n+1}=(1-\lambda) x_{n}+\lambda y_{n},
$$

where $y_{n} \in T x_{n}$ and $\lambda \in(0,1-k)$. Then, $\lim _{n \rightarrow \infty} d\left(x_{n}, T x_{n}\right)=0$.

The result of Chidume et al. (2013) is more interesting than other similar result in the literature because it deals with strictly pseudocontractive mappings(which is more general than nonexpansive mappings) and also the problem of finding $z_{n} \in T x_{n}$ such that $\left\|z_{n}-x^{*}\right\|=d\left(x^{*}, T x_{n}\right)$ as it is, for example, in Sastry and Babu (2005), does not arise. However, the inequality (2) is equivalent to

$$
D^{2}(T x, T y) \leq\|x-y\|^{2}+k \inf _{(u, v) \in(T x, T y)}\|(x-u)-(y-v)\|^{2} .
$$

which is very restrictive

Very recently, Chidume and Okpala (2014) introduced a different class of multi-valued strictly pseudocontractive mapping as given below:

Definition 1.4 Chidume and Okpala (2014) Let $H$ be a real Hilbert space and let $K$ be a nonempty subset of $H$. Let $T: K \rightarrow C B(K)$ be a multi-valued mapping. Then $T$ is called generalized $k$-strictly pseudocontractive multi-valued mapping if there exists $k \in(0,1)$ such that for all $x, y \in D(T)$, there holds

$$
D^{2}(T x, T y) \leq\|x-y\|^{2}+k D^{2}(A x, A y), \quad \text { where } \quad A:=I-T,
$$

and $I$ is the identity operator on $K$.

The class of mapping introduced here is natural and has been proved to be a proper superset of the class introduced in Chidume et al. (2013).

They developed some new identities regarding Hausdorf metric and used a Krasnoselskii type algorithm and obtained the following theorem.

Theorem 1.5 (Chidume and Okpala (2014)) Let $K$ be a nonempty, closed, convex subset of a real Hilbert space H. Let $T: K \rightarrow C B(K)$ be a generalized $k$-strictly pseudocontractive 
multi-valued mapping such that $F(T) \neq \emptyset$. Assume $T p=\{p\} \forall p \in F(T)$. Define a sequence $\left\{x_{n}\right\}$ by $x_{0} \in K$,

$$
x_{n+1}=(1-\lambda) x_{n}+\lambda y_{n}
$$

for $y_{n} \in U^{n}$ and $\lambda \in(0,1-k)$. Then, $d\left(x_{n}, T x_{n}\right) \rightarrow 0$ as $n \rightarrow \infty$, where

$$
U^{n}:=\left\{y_{n} \in T x_{n}: D^{2}\left(\left\{x_{n}\right\}, T x_{n}\right) \leq\left\|x_{n}-y_{n}\right\|^{2}+\frac{1}{n^{2}}\right\} .
$$

We seek to prove strong convergence theorems, using a new averaged algorithm, for common fixed point of a countably infinite family of this general class of mappings in a real Hilbert space. Our theorem generalizes the results of Chidume et al. (2013), Chidume and Ezeora (2014), Panyanak (2007), Song and Wang (2008), among others and extends to a countable family the results of Chidume and Okpala (2014).

\section{Preliminaries}

We shall need the following definitions and notations in the sequel:

We casually denote $(D(A, B))^{2}$ by $D^{2}(A, B)$ for all $A, B \in C B(X)$ for simplicity of notation.

Definition 2.1 A multi-valued mapping $T: K \subseteq H \rightarrow C B(H)$ is called

- Lipschitzian if there exists $L>0$ such that for each $x, y \in K$,

$$
D(T x, T y) \leq L\|x-y\|,
$$

- nonexpansive if there exist $L \leq 1$ such that $T$ is Lipschitchitzian.

Proposition 2.2 (Chidume and Okpala (2014)) Let $K$ be a nonempty subset of a real Hilbert space $H$ and $T: K \rightarrow C B(K)$ be a generalized $k$-strictly pseudocontractive multivalued mapping. Then $T$ is Lipschitzian.

Remark 2.3 Since every Lipschitz map is continuous, we would not make any continuity assumption on our mapping $T$ throughout this paper.

Definition 2.4 A map $T: K \rightarrow C B(K)$ is said to be hemicompact if, for any sequence $\left\{x_{n}\right\}$ such that $\lim _{n \rightarrow \infty} d\left(x_{n}, T x_{n}\right)=0$, there exists a subsequence, say, $\left\{x_{n_{k}}\right\}$ of $\left\{x_{n}\right\}$ such that $x_{n_{k}} \rightarrow p \in K$.

Remark 2.5 Trivial example of hemicompact mappings are mapping with compact domains.

Definition 2.6 Let $H$ be a real Hilbert space and let $T$ be a multi-valued mapping. The multi-valued mapping $I-T$ is said to be strongly demiclosed at 0 (see, e.g., Garcí a-Falset et al. (2011)) if for any sequence $\left\{x_{n}\right\} \subseteq D(T)$ such that $x_{n} \rightarrow p$ and $d\left(x_{n}, T x_{n}\right)$ converges strongly to 0 , then $d(p, T p)=0$. 
Proposition 2.7 (Chidume and Okpala (2014)) Let $K$ be a nonempty and closed subset of a real Hilbert space $H$ and let $T: K \rightarrow C B(K)$ be a generalized $k$-strictly pseudocontractive multi-valued mapping. Then, $(I-T)$ is strongly demiclosed at zero.

The following recurrent inequality will be used to make estimates in the sequel.

Lemma 2.8 (Tan and $\mathrm{Xu}(1993))$ Let $\left\{a_{n}\right\}$ be a sequence of nonnegative real numbers satisfying the following relation:

$$
a_{n+1} \leq a_{n}+\sigma_{n}, \quad n \geq 0,
$$

such that $\sum_{n=1}^{\infty} \sigma_{n}<\infty$. Then, $\lim a_{n}$ exists. If, in addition, $\left\{a_{n}\right\}$ has a subsequence that converges to 0 , then $a_{n}$ converges to 0 as $n \rightarrow \infty$.

Lemma 2.9 (Chidume and Ezeora (2014)) Let $H$ be a real Hilbert space and let $\left\{x_{i}, i=1,2, \ldots, m\right\} \subseteq H$. For $\alpha_{i} \in(0,1), i=1,2, \ldots$, m such that $\sum_{i=1}^{m} \alpha_{i}=1$, the following identity holds:

$$
\left\|\sum_{i=1}^{m} \alpha_{i} x_{i}\right\|^{2}=\sum_{i=1}^{m} \alpha_{i}\left\|x_{i}\right\|^{2}-\sum_{1 \leq i<j \leq m} \alpha_{i} \alpha_{j}\left\|x_{i}-x_{j}\right\|^{2},
$$

The following characterizations of the Hausdorf metric can be found in Chidume and Okpala (2014).

Lemma 2.10 (Chidume and Okpala (2014)) Let $E$ be a normed linear space, $B_{1}, B_{2} \in C B(E)$ and $x, y \in E$ arbitrary. The following hold;

(a) $D\left(B_{1}, B_{2}\right)=D\left(x+B_{1}, x+B_{2}\right)$. Translation Invariance.

(b) $D\left(B_{1}, B_{2}\right)=D\left(-B_{1},-B_{2}\right)$.

(c) $D\left(x+B_{1}, y+B_{2}\right) \leq\|x-y\|+D\left(B_{1}, B_{2}\right)$. Triangle inequality.

(d) $D\left(\{x\}, B_{1}\right)=\sup _{b_{1} \in B_{1}}\left\|x-b_{1}\right\|$.

(e) $D\left(\{x\}, B_{1}\right)=D\left(0, x-B_{1}\right)$.

\section{Fixed point iterations}

The example given below shows that this general class of $k$-strictly pseudocontractive mappings actually exists and properly contains the class studied by Chidume et al. (2013), Osilike and Isiogugu (2011), Panyanak (2007), and a host of other authors. For the example, we shall need the following lemma, which is easy to verify.

Lemma 3.1 Let $a, b$, $c$ be real numbers such that $0 \leq a \leq b c, c>0$. Then

$$
(a-b)^{2} \leq b^{2}+\left(\frac{c-2}{c}\right) a^{2} .
$$


Remark 3.2 By setting $c=4$ in the lemma above, we will recover Lemma (3.5) of Chidume and Okpala (2014).

Example 3.3 Define a multi-valued mapping $T_{i}: l_{2}(\mathbb{R}) \rightarrow C B\left(l_{2}(\mathbb{R})\right)$ by

$$
T_{i} x:=\left\{\begin{array}{l}
\left\{y \in l_{2}:\|x+y\| \leq \alpha_{i}\|x\|\right\}, \quad x \neq 0 \\
\{0\}, \quad x=0,
\end{array}\right.
$$

where $\alpha_{i}=\frac{7 i}{3 i-1}, i=1,2, \ldots$, We obtain that

$$
x-T_{i} x:=\left\{\begin{array}{l}
\left\{y \in l_{2}:\|y-2 x\| \leq \alpha_{i}\|x\|\right\}, \quad x \neq 0 \\
\{0\}, \quad x=0
\end{array}\right.
$$

Then, for arbitrary $x, y \in l_{2}(\mathbb{R})$, we compute as follows:

$$
D\left(T_{i} x, T_{i} y\right)=\|x-y\|+\alpha_{i}|\|x\|-\|y\||,
$$

and

$$
D\left(x-T_{i} x, y-T_{i} y\right)=2\|x-y\|+\alpha_{i}|\|x\|-\|y\|| .
$$

Now, set

$$
a:=D\left(x-T_{i} x, y-T_{i} y\right) ; \quad b:=\|x-y\| .
$$

Then, $a-b=D\left(T_{i} x, T_{i} y\right)$ and

$$
\begin{aligned}
a & =2\|x-y\|+\alpha_{i}|\|x\|-\|y\|| \\
& \leq\left(2+\alpha_{i}\right)\|x-y\| .
\end{aligned}
$$

Now, for each $i$, set $2+\alpha_{i}=c_{i}=c$ in Lemma (3.1) above. We obtain the identity $\frac{c_{i}-2}{c_{i}}=\frac{\alpha_{i}}{2+\alpha_{i}}$, and by the same lemma, we have

$$
D^{2}\left(T_{i} x, T_{i} y\right) \leq\|x-y\|^{2}+\frac{\alpha_{i}}{2+\alpha_{i}} D\left(x-T_{i} x, y-T_{i} y\right)
$$

Thus, each $T_{i}, i=1,2, \ldots$, is a generalized $\kappa_{i}$-strictly pseudo-contractive multi-valued mapping with $\kappa_{i}=\frac{\alpha_{i}}{2+\alpha_{i}} \in(0,1)$ and each $\kappa_{i} \leq \kappa:=\frac{7}{13}$. Moreover, we have $p \in T_{i} p$ if and only if $p=0$. Thus, for $p \in \cap_{i=1}^{\infty} F\left(T_{i} p\right), T_{i} p=\{p\}$.

The following Lemma would be used in the sequel.

Lemma 3.4 Let $H$ be a real Hilbert space and let $\left\{x_{i}\right\}_{i \in \mathbb{N}}$ be a bounded sequence in $H$. For $\delta_{i} \in(0,1)$, such that $\sum_{i=1}^{\infty} \delta_{i}=1$, the following identity holds:

$$
\left\|\sum_{i=1}^{\infty} \delta_{i} x_{i}\right\|^{2}=\sum_{i=1}^{\infty} \delta_{i}\left\|x_{i}\right\|^{2}-\sum_{1 \leq i<j<\infty} \delta_{i} \delta_{j}\left\|x_{i}-x_{j}\right\|^{2} .
$$


Proof Define $\delta_{i}(n):=\left(1-\sum_{n+1}^{\infty} \delta_{i}\right)^{-1} \delta_{i}$ for each $n$. It is easily seen that $\sum_{i=1}^{n} \delta_{i}(n)=1$ and that $\delta_{i}(n) \rightarrow \delta_{i}$ as $n \rightarrow \infty$. Moreover, by Lemma 2.9, we obtain that

$$
\| \sum_{i=1}^{n} \delta_{i}\left((n) x_{i}\left\|^{2}=\sum_{i=1}^{n} \delta_{i}(n)\right\| x_{i}\left\|^{2}-\sum_{1 \leq i<j<\leq n} \delta_{i}(n) \delta_{j}(n)\right\| x_{i}-x_{j} \|^{2}\right.
$$

Since the inequality is true for all natural numbers $n$, we pass to the limit on both sides and obtain the identity (10) as proposed.

Next, given a countably infinite family $\left\{T_{i}\right\}_{i \geq 1}$ of generalized $\kappa_{i}$-strictly pseudo-contractive multi-valued mappings and an arbitrary sequence $\left\{x_{n}\right\}$ subset of $K$, denote by $\Gamma_{n}^{i}$ the set of inexact distal points of $x_{n}$ with respect to the set $T_{i} x_{n}$, i.e

$$
\Gamma_{n}^{i}:=\left\{\zeta_{n}^{i} \in T_{i} x_{n}: D^{2}\left(\left\{x_{n}\right\}, T_{i} x_{n}\right) \leq\left\|x_{n}-\zeta_{n}^{i}\right\|^{2}+\frac{1}{n^{2}}\right\}
$$

Obviously, $\Gamma_{n}^{i}$ is closed, convex and nonempty for each $n \geq 1$ due to Lemma (2.10)(d).

In particular, if $T_{i} x$ is assumed to be proximinal and bounded for each $x \in K$, then $T_{i} x_{n}$ has a vector, say $\eta_{n}^{i}$, of maximum norm, i.e.

$$
\left\|x_{n}-\eta_{n}^{i}\right\|=\sup _{\zeta_{n}^{i} \in T_{i} x_{n}}\left\|x_{n}-\zeta_{n}^{i}\right\|=: D\left(\left\{x_{n}\right\}, T_{i} x_{n}\right) .
$$

In that case, it is certain that $\eta_{n}^{i} \in \Gamma_{n^{*}}^{i}$

Based upon these analyses, we now prove our main theorem. We will assume henceforth that $K$ is a nonempty, closed and convex subset of a real Hilbert space $H$.

Theorem 3.5 Let $T_{i}: K \rightarrow C B(K)$ be a countably infinite family of generalized $\kappa_{i}$-strictly pseudocontractive multi-valued mappings such that for some $\kappa \in(0,1), \kappa_{i} \in(0, \kappa]$. Assume that $\cap_{i=1}^{\infty} F\left(T_{i}\right) \neq \emptyset$ and for $p \in \cap_{i=1}^{\infty} F\left(T_{i}\right), T_{i} p=\{p\}$. Define the sequence $\left\{x_{n}\right\}$ recursively by

$$
\left\{\begin{array}{l}
x_{0} \in K, \text { arbitrary, } \\
\zeta_{n}^{i} \in \Gamma_{n}^{i}, \\
x_{n+1}=\delta_{0} x_{n}+\sum_{i=1}^{\infty} \delta_{i} \zeta_{n}^{i}, \\
\delta_{0} \in(\kappa, 1), \quad \sum_{i=0}^{\infty} \delta_{i}=1 .
\end{array}\right.
$$

Then, for each $i, \lim _{n \rightarrow \infty} d\left(x_{n}, T_{i} x_{n}\right)=0$.

Proof We will first of all establish that the recursion formula $x_{n+1}:=\delta_{0} x_{n}+\sum_{i=1}^{\infty} \delta_{i} \zeta_{n}^{i}$ in the algorithm (11) is well defined. Take $p \in \cap_{i=1}^{\infty} F\left(T_{i}\right)$ arbitrary. We have

$$
\begin{aligned}
\left\|x_{n}-\zeta_{n}^{i}\right\| & \leq D\left(x_{n}, T_{i} x_{n}\right), \\
& =D\left(x_{n}+p, p+T_{i} x_{n}\right) .
\end{aligned}
$$


Therefore, we obtain by Lemma $2.10(\mathrm{c})$ that

$$
\begin{aligned}
\left\|x_{n}-\zeta_{n}^{i}\right\| & \leq\left\|x_{n}-p\right\|+D\left(T p, T_{i} x_{n}\right) \\
& \leq\left\|x_{n}-p\right\|+\frac{1+\sqrt{\kappa}}{1-\sqrt{\kappa}}\left\|x_{n}-p\right\| .
\end{aligned}
$$

As a matter of fact, we may apply the triangle inequality and take limits to obtain

$$
\left\|\zeta_{n}^{i}\right\| \leq K_{n}:=\left\|x_{n}\right\|+\frac{2}{1-\sqrt{\kappa}} \inf _{p \in F(T)}\left\|x_{n}-p\right\| .
$$

It follows then that

$$
\left\|x_{n+1}\right\| \leq \delta_{0}\left\|x_{n}\right\|+\sum_{i=1}^{\infty} \delta_{i}\left\|\zeta_{n}^{i}\right\|
$$

and therefore

$$
\left\|x_{n+1}\right\| \leq \delta_{0}\left\|x_{n}\right\|+\sum_{i=1}^{\infty} \delta_{i} K_{n} \leq K_{n}
$$

which shows that $x_{n+1}$ is well defined. We show the convergence of $\left\{x_{n}\right\}$ as follows:

Since $\zeta_{n}^{i} \in \Gamma_{n}^{i}$, we obtain that

$$
\begin{aligned}
\left\|x_{n+1}-p\right\|^{2} & =\left\|\delta_{0}\left(x_{n}-p\right)+\sum_{i=1}^{\infty} \delta_{i}\left(\zeta_{n}^{i}-p\right)\right\|^{2} \\
& =\delta_{0}\left\|x_{n}-p\right\|^{2}+\sum_{i=1}^{\infty} \delta_{i}\left\|\zeta_{n}^{i}-p\right\|^{2}-\sum_{i=1}^{\infty} \delta_{0} \delta_{i}\left\|x_{n}-\zeta_{n}^{i}\right\|^{2}-\sum_{1 \leq i \leq j \leq \infty} \delta_{i} \delta_{j}\left\|\zeta_{n}^{i}-\zeta_{n}^{j}\right\|^{2} \\
& \leq \delta_{0}\left\|x_{n}-p\right\|^{2}+\sum_{i=1}^{\infty} \delta_{i} D^{2}\left(T_{i} x_{n}, T p\right)-\sum_{i=1}^{\infty} \delta_{0} \delta_{i}\left\|x_{n}-\zeta_{n}^{i}\right\|^{2} \\
& \leq \delta_{0}\left\|x_{n}-p\right\|^{2}+\sum_{i=1}^{\infty} \delta_{i}\left(\left\|x_{n}-p\right\|^{2}+\kappa_{i} D^{2}\left(\{0\}, x_{n}-T_{i} x_{n}\right)\right)-\sum_{i=1}^{\infty} \delta_{0} \delta_{i}\left\|x_{n}-\zeta_{n}^{i}\right\|^{2} \\
& =\sum_{i=0}^{\infty} \delta_{i}\left\|x_{n}-p\right\|^{2}+\sum_{i=1}^{\infty} \delta_{i} \kappa_{i} D^{2}\left(\left\{x_{n}\right\}, T_{i} x_{n}\right)-\sum_{i=1}^{\infty} \delta_{0} \delta_{i}\left\|x_{n}-\zeta_{n}^{i}\right\|^{2} \\
\left\|x_{n+1}-p\right\| & \leq \sum_{i=0}^{\infty} \delta_{i}\left\|x_{n}-p\right\|^{2}+\sum_{i=1}^{\infty} \delta_{i} \kappa\left(\left\|x_{n}-\zeta_{n}^{i}\right\|^{2}+\frac{1}{n^{2}}\right)-\sum_{i=1}^{\infty} \delta_{0} \delta_{i}\left\|x_{n}-\zeta_{n}^{i}\right\|^{2} \\
& \leq\left\|x_{n}-p\right\|^{2}+\frac{\kappa}{n^{2}}-\sum_{i=1}^{\infty} \delta_{i}\left(\delta_{0}-k\right)\left(\left\|x_{n}-\zeta_{n}^{i}\right\|^{2}\right) .
\end{aligned}
$$

This is summarised as:

$$
\left\|x_{n+1}-p\right\|^{2} \leq\left\|x_{n}-p\right\|^{2}+\frac{\kappa}{n^{2}}-\sum_{i=1}^{\infty} \delta_{i}\left(\delta_{0}-\kappa\right)\left\|x_{n}-\zeta_{n}^{i}\right\|^{2},
$$


and therefore

$$
\left\|x_{n+1}-p\right\|^{2} \leq\left\|x_{n}-p\right\|^{2}+\frac{\kappa}{n^{2}} .
$$

In accordance with Lemma (2.8), $\left\|x_{n}-p\right\|$ has a limit and thus $\left\{x_{n}\right\}$ is bounded. Also, from inequality (12), there holds:

$$
\sum_{i=1}^{\infty} \delta_{i}\left(\delta_{0}-\kappa\right)\left\|x_{n}-\zeta_{n}^{i}\right\|^{2} \leq\left\|x_{n}-p\right\|^{2}+\frac{\kappa}{n^{2}}-\left\|x_{n+1}-p\right\|^{2}
$$

and so for each $i \geq 1$,

$$
\delta_{i}\left(\delta_{0}-\kappa\right)\left\|x_{n}-\zeta_{n}^{i}\right\|^{2} \leq\left\|x_{n}-p\right\|^{2}+\frac{\kappa}{n^{2}}-\left\|x_{n+1}-p\right\|^{2}, \rightarrow 0(\text { as } n \rightarrow \infty),
$$

Taking limits on both sides as $n \rightarrow \infty$, we conclude that $\lim _{n \rightarrow \infty}\left\|x_{n}-\zeta_{n}^{i}\right\|=0$. Using the fact that $d\left(x_{n}, T_{i} x_{n}\right) \leq\left\|x_{n}-\zeta_{n}^{i}\right\|$, we get $\lim _{n \rightarrow \infty} d\left(x_{n}, T_{i} x_{n}\right)=0$.

Corollary 3.6 Let $T_{i}: K \rightarrow C B(K)$ be a countably infinite family of generalized $\kappa_{i}$-strictly pseudocontractive multi-valued mappings such that for some $\kappa \in(0,1), \kappa_{i} \in(0, \kappa]$. Assume that $\cap_{i=1}^{\infty} F\left(T_{i}\right) \neq \emptyset$ and suppose that for $p \in \cap_{i=1}^{\infty} F\left(T_{i}\right)$, $T_{i} p=\{p\}$. Assume $T_{i_{0}}$ is hemicompact for some $i_{0}$. Then, the sequence $\left\{x_{n}\right\}$ defined by algorithm (11) converges strongly to a fixed point of $T$.

Proof We already have that $\lim _{n \rightarrow \infty} d\left(x_{n}, T_{i} x_{n}\right)=0$ due to Theorem (3.5). The mapping $T_{i_{0}}$ being hemicompact guarantees the existence of some subsequence, say $\left\{x_{n_{k}}\right\}$, of $\left\{x_{n}\right\}$ such that $x_{n_{k}} \rightarrow q$ as $k \rightarrow \infty$. Let $\zeta_{n_{k}}^{i} \in T_{i} x_{n_{k}}$ be such that $\left\|x_{n_{k}}-\zeta_{n_{k}}^{i}\right\| \leq d\left(x_{n_{k}}, T_{i} x_{n_{k}}\right)+\frac{1}{k}$. We estimate that

$$
\begin{aligned}
d\left(q, T_{i} q\right) & \leq\left\|q-x_{n_{k}}\right\|+\left\|x_{n_{k}}-\zeta_{n_{k}}^{i}\right\|+d\left(\zeta_{n_{k}}^{i}, T_{i} q\right) \\
& \leq\left\|q-x_{n_{k}}\right\|+d\left(x_{n_{k}}, T_{i} x_{n_{k}}\right)+\frac{1}{k}+D\left(T_{i} x_{n_{k}}, T_{i} q\right) \\
& \leq\left\|q-x_{n_{k}}\right\|+d\left(x_{n_{k}}, T_{i} x_{n_{k}}\right)+\frac{1}{k}+\frac{1+\sqrt{\kappa}}{1-\sqrt{\kappa}}\left\|x_{n_{k}}-q\right\| .
\end{aligned}
$$

If we take limits on both sides when $k \rightarrow \infty$, we have $d\left(q, T_{i} q\right)=0$. Using the fact that each $T_{i} q$ is closed, we obtain that $q \in T_{i} q$ for each $i$, and therefore conclude that $q \in \cap_{i=1}^{\infty} T_{i} q$. Moreover, $x_{n_{k}} \rightarrow q$ as $n \rightarrow \infty$ gives $\left\|x_{n_{k}}-q\right\| \rightarrow 0$ as $n \rightarrow \infty$. Thus, by Lemma (2.8) and inequality (13), we get $\lim _{n \rightarrow \infty}\left\|x_{n}-q\right\|=0$. Thus $\left\{x_{n}\right\}$ converges strongly to a fixed point $q$ of $T$ as claimed.

Corollary 3.7 Let $T_{i}: K \rightarrow C B(K)$ be a countably infinite family of generalized $\kappa_{i}$-strictly pseudocontractive multi-valued mapping, with $\cap_{i=1}^{\infty} F\left(T_{i}\right) \neq \emptyset$ and assume that for $p \in \cap_{i=1}^{\infty} F\left(T_{i}\right), T_{i} p=\{p\}$. Then, the sequence $\left\{x_{n}\right\}$ defined by Eq. (11) converges strongly to a fixed point of $T$. 
Proof Since $K$ is compact, the mappings $T_{i}: K \rightarrow C B(K)$ is hemicompact. Thus, by Corollary (3.6), we have that $\left\{x_{n}\right\}$ converges strongly to some $p \in F(T)$.

Remark 3.8 In comparison with Theorem 7.1.5 of Chidume and Ezeora (2014), Corollary 3.6 has these merits.

(i) We proved the theorem for a countably infinite family of a much larger class of mapping which is the generalized $k$-strictly pseudo-contractive multi-valued mappings.

(ii) We only needed just one of the maps to be hemicompact and not all of them.

(iii) We replaced the 'strong condition' $\delta_{i} \in(k, 1)$ by a weaker condition $\delta_{0} \in(k, 1)$.

(iv) The condition $\zeta_{n}^{i} \in \Gamma_{n}^{i}$ is more readily applicable than requiring that $T x$ is proximinal and weakly closed for each $x$, and then, computing $\zeta_{n}=P_{T x_{n}} x_{n}$ at each iterative step.

\section{Conclusion}

Our theorem and corollaries improve the convergence theorems for multi-valued nonexpansive mappings in Abbas et al. (2011), Chidume et al. (2013), Chidume and Ezeora (2014), Chidume and Okpala (2014), Khan and Yildirim (2012), Ofoedu and Zegeye (2010), Panyanak (2007), Sastry and Babu (2005), Song and Wang (2008), in the following sense:

(i) The class of mappings considered in this paper contains the class of multi-valued $k$-strictly pseudocontractive mappings as a special case, which itself properly contain the class of multi-valued nonexpansive maps.

(ii) The algorithm here is of Krasnoselkii type, which is known to have a geometric order of convergence.

(iii) The condition that $T x$ be weakly closed for each $x \in K$ as can be found, for example, in Chidume et al. (2013) and Chidume and Ezeora (2014) is dispensed with here.

Authors' contributions

CE proposed the problem. ME worked out the intricacies and drafted the first version of the manuscript. Both authors read and approved the final manuscript.

\section{Author details}

${ }^{1}$ Mathematics Institute, African University of Sciences and Technology, Abuja, Nigeria. ${ }^{2}$ Department of Mathematics, Federal University Ndufu-Alike Ikwo, Abakaliki, Ebonyi State, Nigeria.

\section{Acknowledgements}

The authors would like to acknowledge funding from the African Capacity Building Foundation (ACBF) for the publication of this manuscript.

\section{Compliance with ethical guidelines}

Competing interests

The authors declare that they have no competing interests.

Received: 22 July 2015 Accepted: 27 August 2015

Published online: 17 September 2015

\section{References}

Abbas M, Khan SH, Khan AR, Agarwal RP (2011) Common fixed points of two multi-valued nonexpansive mappings by onestep iterative scheme. Appl Math Lett 24(2):97102 
Brouwer LEJ (1912) Uber Abbildung von Mannigfaltigkeiten. Math Ann 71(4):598

Browder FE, Petryshyn WE (1967) Construction of fixed points of nonlinear mappings in Hilbert space. J Math Anal Appl 20:197-228

Browder FE (1967) Nonlinear mappings of nonexpansive and accretive type in Banach Spaces. Bull Am Math Soc 73:875-882

Chidume CE, Chidume CO, Djitte N, Minjibir MS (2013) Convergence theorems for fixed points of multi-valued strictly pseudocontractive mappings in Hilbert spaces. Abstr Appl Anal 2013:629468. doi:10.1155/2013/629468

Chidume CE, Ezeora JN (2014) Krasnoselskii-type algorithm for family of multi-valued strictly pseudo-contractive mappings. Fixed Point Theory Appl 111. doi:10.1186/1687-1812-2014-111

Chidume CE, Okpala ME (2014) On a general class of multi-valued strictly pseudocontractive mappings. J Nonlinear Anal Optim Theory Appl 5(2):7-20

Daffer PZ, Kaneko H (1995) Fixed points of generalized contractive multi-valued mappings. J Math Anal Appl 192(2):655666

Deimling K (1992) Multi-valued differential equations, vol 1. Walter de Gruyter \& Co., Berlin

Downing D, Kirk WA (1977) Fixed point theorems for set-valued mappings in metric and Banach spaces. Math Jpn 22(1):99-112

García-Falset J, Lorens-Fuster E, Suzuki T (2011) Fixed point theory for a class of generalized nonexpansivemappings. J Math Anal Appl 375(1):185-195

Geanakoplos J (2003) Nash and walras equilibrium via Brouwer. Econ Theory 21:585-603

Kakutani S (1941) A generalization of Brouwer's fixed point theorem. Duke Math J 8(3):457-459

Khan SH, Yildirim I (2012) Fixed points of multi-valued nonexpansive mappings in Banach spaces. Fixed Point Theory Appl 2012, article 73

Markin JT (1973) Continuous dependence of fixed point sets. Proc Am Math Soc 38:545547

Nadler SB Jr (1969) Multi-valued contraction mappings. Pac J Math 30:475-488

Nash JF (1950) Equilibrium points in n-person games. Proc Natl Acad Sci USA 36(1):48-49

Nash JF (1951) Non-cooperative games, second series. Ann Math 54:286-295

Ofoedu EU, Zegeye H (2010) Iterative algorithm for multi-valued pseudocontractive mappings in Banach spaces. J Math Anal Appl 372:68-76

Osilike MO, Isiogugu FO (2011) Weak and strong convergence theorems for nonspreading-type mappings in Hilbert spaces. Nonlinear Anal 74:18141822

Panyanak B (2007) Mann and Ishikawa iterative processes for multi-valued mappings in Banach spaces. Comput Math Appl 54(6):872-877

Reich S, Zaslavski AJ (2002) Convergence of iterates of nonexpansive set-valued mappings in set valued mappings with applications in nonlinear analysis. Math Anal Appl Taylor Francis Lond UK 4:411420

Reich S, Zaslavski AJ (2002) Generic existence of fixed points for set-valued mappings. Set Valued Anal 10(4):287296

Reich S, Zaslavski AJ (2006) Two results on fixed points of set-valued nonexpansive mappings. Revue Roumaine de Mathematiques Pures et Appliquees 51(1):8994

Sastry KPR, Babu GVR (2005) Convergence of Ishikawa iterates for a multi-valued mapping with a fixed point. Czechoslov Math J 55(4):817826

Song Y, Wang H (2008) Erratum to; Mann and Ishikawa iterative processes for multi-valued mappings in Banach Spaces [Comput. Math. Appl. 54 (2007), 872877]. Comput Math Appl 55:2999-3002

Tan KK, Xu HK (1993) Approximating fixed points of nonexpansive mappings by the Ishikawa Iteration Process. J Math Anal Appl 178(2):301-308

\section{Submit your manuscript to a SpringerOpen ${ }^{\circ}$ journal and benefit from:}

- Convenient online submission

- Rigorous peer review

- Immediate publication on acceptance

- Open access: articles freely available online

- High visibility within the field

- Retaining the copyright to your article

Submit your next manuscript at $\boldsymbol{s p r i n g e r o p e n . c o m ~}$ 